\title{
Taxonomy of trans-Neptunian objects and Centaurs as seen from spectroscopy $\star$
}

\author{
F. Merlin ${ }^{1}$, T. Hromakina ${ }^{2}$, D. Perna ${ }^{1}$, M. J. Hong ${ }^{1}$, and A. Alvarez-Candal ${ }^{3}$ \\ ${ }^{1}$ LESIA - Observatoire de Paris, PSL Research University, CNRS, Sorbonne Universités, UPMC Univ. Paris 06, Univ. Paris Diderot, \\ Sorbonne Paris Cité, 5 place Jules Janssen, 92195 Meudon, France \\ e-mail: frederic.merlin@obspm.fr \\ 2 Institute of Astronomy, Kharkiv V. N. Karin National University, Sumska Str. 35, 61022 Kharkiv, Ukraine \\ 3 Observatorio Nacional, R. Gal. Jose Cristino 77, 20921-400 Rio de Janeiro, Brazil
}

Received 4 April 2017 / Accepted 19 May 2017

\begin{abstract}
Context. Taxonomy of trans-Neptunian objects (TNOs) and Centaurs has been made in previous works using broadband filters in the visible and near infrared ranges. This initial investigation led to the establishment of four groups with the aim to provide the mean colors of the different classes with possible links with any physical or chemical properties. However, this taxonomy was only made with the Johnson-Cousins filter system and the ESO $J, H, K$ s filters combination, and any association with other filter system is not yet available.

Aims. We aim to edit complete visible to near infrared taxonomy and extend this work to any possible filters system. To do this, we generate mean spectra for each individual group, from a data set of 43 spectra. This work also presents new spectra of the TNO (38628) Huya, on which aqueous alteration has been suspected, and the Centaur $2007 \mathrm{VH}_{305}$.

Methods. To generate the mean spectra for each taxonomical group, we first averaged the data for each of the four taxonomical groups and checked that spectroscopic and photometric data were consistent according to their relative errors.

Results. We obtained four complete spectra corresponding to the different classes from 0.45 to 2.40 microns. Our results based on spectroscopy are in good agreements with those obtained in photometry for the bluest (BB) and reddest (RR) objects. At the contrary, no clear patterns appear for the two intermediate groups (BR and IR). Both BR and IR mean-spectra are almost intermixed, probably due to the fact that part of these objects have not always clear affiliation to one particular taxonomical group.

Conclusions. We provide mean spectra that could be used to edit colors in different filters system working in this wavelength range. This work clearly establish the mean spectra of the BB and RR group while the two other groups need probably further refinement.
\end{abstract}

Key words. Kuiper belt: general - Kuiper belt objects: individual: (38628) Huya - minor planets, asteroids: individual: 2007 VH305 - techniques: spectroscopic

\section{Introduction}

In the last decades the outer solar system has been found to be densely populated by many icy bodies called trans-Neptunian objects (TNOs). These faint and distant objects are supposed to be the remnants of the proto-planetary disk, and give us hints on the mechanisms which governed the evolution of the early solar nebula as well as of other planetary systems around young stars (see Barucci et al. 2008a, for an introduction on TNOs overview). More than 1500 TNOs are known up to today with well-constrained orbits, presenting different sizes, orbits and surface characteristics. The availability of very large $(8-10 \mathrm{~m})$ ground-based telescopes, as well as of Earth-orbiting facilities (Hubble, Spitzer, Herschel), has enabled observational studies of physical properties of a significant number of objects, but the physical knowledge of smaller objects still remains poor. Obtaining this information for the whole population is still a big challenge and is mostly limited to the photometrical approach.

Broadband photometry represents the simplest observing technique to study physical properties of TNOs and Centaurs and color data provide an accurate measure of the global surface reflectance of the objects. Doressoundiram et al. (2008) show that there is no doubt that a large number of TNOs and Centaurs have

\footnotetext{
* Part of the data presented here have been performed under ESO Programmes ID 178.C-0867 and ID 091.C-0224.
}

very red surface colors, in fact much redder than other solar system bodies but objects with neutral colors are also found. Their statistical analyses point to correlations between optical colors and orbital inclination and orbital rms velocity for the classical objects (see the review made by Gladman et al. 2008, for a detailed summary of the different dynamical families). On the other hand, they do not point any clear trend for Plutinos, scattered objects or Centaurs, and correlation of colors with size or heliocentric distances neither. On the basis of the present available data sample, a huge diversity is evident among TNOs. Spectral data show a wide diversity in terms of different general behaviors (from blue to very red) as well as component signatures (from featureless, to different contents of ices). This color or spectral variegation appears to be the results of different processes such as collision, space weathering, outgassing, impact gardening, that work at different levels on the surface of these minor bodies (Barucci et al. 2008b).

As color investigation fail to identify clear trends among the TNOs and Centaurs populations, spectroscopic investigation was performed in attempt to obtain chemical informations of the surface of these minor bodies, and investigate the possibility to link, with this more accurate tool, correlations between physical and chemical properties (Barucci et al. 2011). From a total sample of 75 objects, they carried out analyses on the ice content with respect to the physical and dynamical characteristics. Their work 
show that all objects exhibiting neutral or slightly blue visible spectral slope seem to have icy surfaces, that the possible presence of $\mathrm{CH}_{3} \mathrm{OH}$ has primarily been detected on very red surfaces and that the majority of Centaurs observed multiple times have an heterogeneous composition.

Since spectroscopy is limited to the brightest objects (that are usually the biggest or closest), Barucci et al. (2005) and Fulchignoni et al. (2008) investigate the color variegation of these objects using statistical tool, in order to derive general trends among the whole TNOs and Centaurs population, and including smaller objects than those that could be observed by spectroscopy. Their statistical work, based on the G-mode analysis (see Barucci et al. 1987, for a more detail explanation of this technique in planetology cases), led to the presence of four distinct taxonomical groups, from the bluest objects (the BB group) to the reddest ones (the RR group) with two intermediate groups (BR and IR). DeMeo et al. (2009) and Perna et al. (2010, 2013) extend this work using new photometry observation carried out in the whole visible-near infrared (nIR) spectral range. These authors looked for correlations between the taxonomic classification and orbital parameters of TNOs and Centaurs, to constrain their formation region and evolution, for example supporting an evolutionary origin for the color dichotomy (BR/RR) of Centaurs. However, clear trends are not really found even if they mainly report that $\mathrm{RR}$ and $\mathrm{BB}$ classes are more abundant at low and high orbital inclinations, respectively, which associates these objects with the dynamically "cold" and "hot" populations. They also found that BR and RR classes dominate among the population at small values of semi-major axis (lower than 30 A.U.), while all the four taxa are well represented at greater distances from the Sun.

Until now, the small tail of this object population could be only investigated by photometry, and that taxonomy could be a valuable proxy to grab chemical information on the $10-100 \mathrm{~km}$ class objects. In the framework of new instruments-telescopes and comparison with different filter sets, a complete visible-nIR investigation is needed and could be only achieved using spectroscopy. In this work, we present first the spectra of two objects, not yet published, and discuss the spectral properties of them. The following section will be devoted to the presentation of the spectroscopic data set used to generate the four different spectra, one for each taxonomical class. Finally we present these four mean spectra in the last section.

\section{New spectra}

In the whole set of spectroscopic data used in this work, there are only two objects for which we obtained new data. The first is the object Huya and the second one is $2007 \mathrm{VH}_{305}$. Data have been collected using two different instruments mounted on the eight-meter class telescopes of the ESO-VLT (X-shooter and SINFONI, respectively).

\subsection{Data of the object Huya}

Observations of Huya have been performed in July and August 2013 using the instrument X-shooter that allows us to obtain a simultaneous spectrum over the entire [0.3-2.4] $\mu \mathrm{m}$ range. Three different spectra have been done in the aim to look at this object at different rotational phases and more especially to investigate any possible absorption features diagnosing aqueous alteration as identified by Lazzarin et al. (2003). We performed eight exposures of $600 \mathrm{~s}$ each the first night and four exposures of $900 \mathrm{~s}$

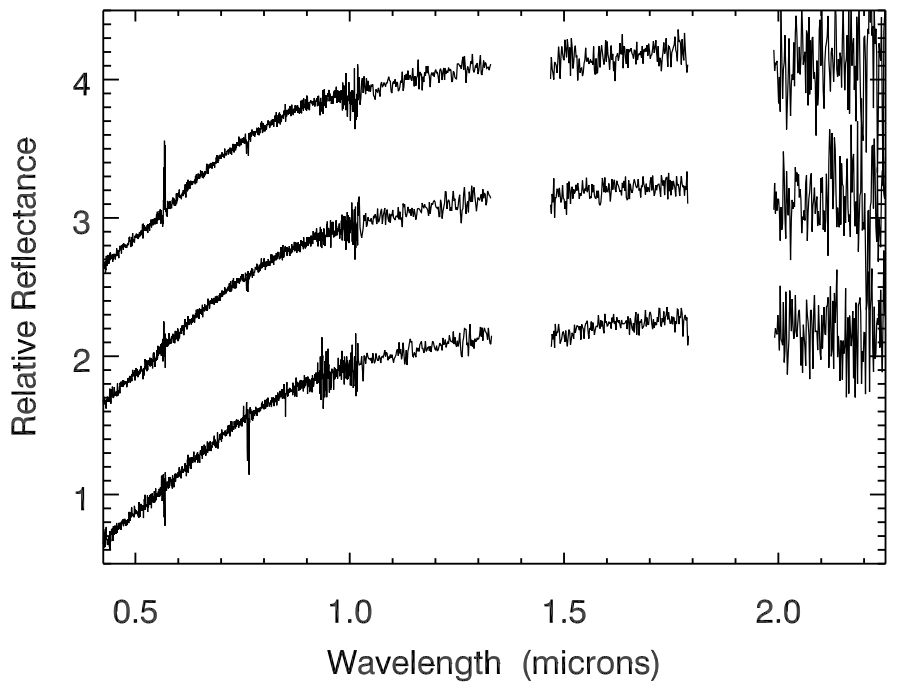

Fig. 1. Three reflectance spectra of Huya, all normalized at $0.55 \mu \mathrm{m}$ and shifted by 0,1 and 2 units, for clarity. Spectra appear chronologically from bottom to the top.

each the last two nights. The detailed description of the instrument is available at ESO's webpage ${ }^{1}$. The observational circumstances are given in Table 1 . We used the SLIT mode, selecting the high-gain readout mode and $2 \times 1$ binning for the UVB and VIS detectors. We nodded on the slit to remove the sky contribution, as usual for NIR observations. The read-out and binning for the NIR detector are fixed. The slit widths were 1.3, 1.2, and 1.2 arcsec for the UVB, VIS, and NIR arms, respectively, yielding a resolving power of at least 4000 per arm.

All data were reduced using Reflex $^{2}$, which automatically performs all calibrations and corrections in each arm (bias or dark current correction, flat-fielding, distortion correction, wavelength calibration, extinction correction, sky extraction, and creation of a 2D image with the spectrum). We then extracted the spectra using IRAF, one per arm, and divided those of Huya by those of the well proven solar analog HD 144585, also used to correct for telluric absorptions in the near-infrared (we verified to obtain consistent results also using the second solar analog, i.e: SA107998). Finally we combined the UVB, VIS, and NIR spectra, thanks to the overlap between the three arms, to produce the final spectra of Huya (normalized to unity at $0.55 \mu \mathrm{m}$ ). We also performed a filtering process with the aim to reduce part of the residual noise. If the value of the spectra at each wavelength is comprised between $\pm 2 \sigma$ around the mean value, both computed from its twenty closest neighbors, then the value is unchanged, else the new value is equal to the mean value. The resulting spectra, spanning the complete range between 350 and $2350 \mathrm{~nm}$ are presented in Fig. 1.

\subsection{Data of the object $2007 \mathrm{VH}_{305}$}

Visible and near-infrared observations have been made within an ESO Large Program. Part of the data have been published for this object. Photometry in both wavelength ranges has been presented in Perna et al. (2010) while results based on visible spectroscopy have been presented in Fornasier et al. (2009). All these data have been done on 22 and 23 November 2008. Spectroscopy

\footnotetext{
1 http://www.eso.org/sci/facilities/paranal/ instruments/xshooter

2 www.eso.org/sci/software/reflex/
} 
Table 1. Observational circumstances.

\begin{tabular}{lcccc}
\hline Object & Date & Time (UT) & Airmass & Solar analog (airmass) \\
\hline Huya & $2013-07-13$ & $23: 44-0: 24$ & $1.07-1.10$ & HD 144585 (1.02) \\
Huya & $2013-07-14$ & $23: 34-0: 34$ & $1.07-1.11$ & HD 144585 (1.02) \\
Huya & $2013-08-04$ & $23: 44-0: 44$ & $1.08-1.16$ & HD 144585 (1.05) \\
2007VH $_{305}$ & $2009-11-23$ & $0: 35-3: 25$ & $1.14-1.24$ & SA93101 (1.17) \\
\hline
\end{tabular}

of the Centaur $2007 \mathrm{VH}_{305}$ in the nIR has not been previously published. NIR spectroscopy was performed within the same ESO Large Program and the observational circumstances are given in Table 1. Seventeen exposures of $600 \mathrm{~s}$ were taken in a jitter pattern, for a total exposure time of $2 \mathrm{~h} 50 \mathrm{~min}$. Solar analog SA93101 (type G5) was observed at an airmass of 1.17. For these observations, the spatial scale was $250 \times 250$ mas. Reduction was performed using the ESO SINFONI pipeline combined with IDL procedures. First the data were corrected for bad lines created from bad pixels located among the four nonilluminated edge pixels at the beginning and end of each row. Master darks, master flats, bad pixel maps, and wave maps were created within the SINFONI pipeline. $\mathrm{Xe}-\mathrm{Ar}-\mathrm{Kr}$ lamps were used for wavelength calibration, and as part of the SINFONI pipeline, a wavelength map was computed to derive a direct correspondence between pixel position and wavelength. Determining the orientation and position of each spectrum on the detector enables the reconstruction of an image-cube of the original field-of-view. The spectra were then extracted from the individual data cubes using QFitsView, the 3D-visualization tool developed at the Max Planck Institute for Extraterrestrial Physics (MPE) for SINFONI ${ }^{3}$. Within QFitsView, an aperture radius and center were chosen and the flux within that circle is summed per image slice to create the spectrum. We chose an aperture size that maximized the flux of the entire disk of the Centaur (as opposed to summing the flux over only a part of the surface) while minimizing the sky background. We finally divided the spectrum of this object by that of the solar analog to produce a reflectance spectrum. Uncertainty in the flux calibration for SINFONI data is less than $5 \%$. The same filtering process as was previously done for the spectra of Huya has been performed too. We merged the different spectra from the visible to the nIR using VJHK colors converted in reflectance values. The spectrum is presented in Fig. 2.

\subsection{Results on the surface properties of the two objects}

Huya belongs to the Plutino family. Its visible albedo is estimated at $8.3 \pm 0.4 \%$ for a diameter of $458 \pm 9.2 \mathrm{~km}$ from thermal observations (Fornasier et al. 2013). However, Noll et al. (2012), discovered the presence of a binary companion 1.4 mag fainter than the primary and new estimations have been established for the diameter of Huya and for that of its satellite $(406 \pm 16 \mathrm{~km}$ and $213 \pm 30 \mathrm{~km}$, respectively).

Huya (formerly known as $2000 \mathrm{~EB}_{173}$ ) has been so far observed by several authors, with very low quality spectra. Jewitt \& Luu (2001) obtained spectrum in the near infrared. The spectrum recorded by Brown et al. (2000) in the 1.4-2.4 $\mu \mathrm{m}$ range is also more of less flat up to $1.9 \mu \mathrm{m}$, but the flux decreases continuously with increasing wavelength due to absorption, which reached approximately $10 \%$ at $2.2 \mu \mathrm{m}$. Licandro et al. (2001) obtained a very red spectrum, whose intensity rises

\footnotetext{
3 http://www.mpe.mpg.de/ ott/QFitsView
}

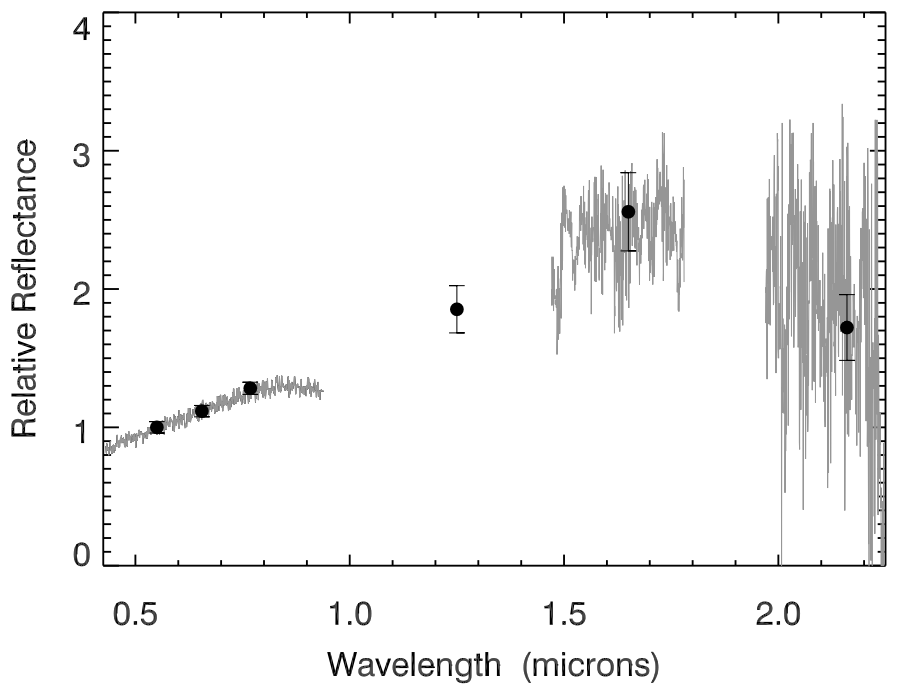

Fig. 2. Reflectance spectrum of $2007 \mathrm{VH}_{305}$ normalized at $0.55 \mu \mathrm{m}$. Photometric data are taken from Perna et al. (2010).

steeply between 0.9 and $1.7 \mu \mathrm{m}$, and detected in a very low quality spectrum a broad absorption in the $K$ band, tentatively assigned to $\mathrm{H}_{2} \mathrm{O}$ ice. Our observations could confirm the presence of $\mathrm{H}_{2} \mathrm{O}$ ice with confirmation of a shallow feature near $2.0 \mu \mathrm{m}$, more obvious on the last two spectra (i.e: obtained in 2013-07-14 and 2013-08-04, see Fig. 1). Alvarez-Candal et al. (2007) confirmed the presence of the absorption feature at $2.0 \mu \mathrm{m}$. Visible spectra of Huya, acquired on April 2001 and May 2002 (Lazzarin et al. 2003; de Bergh et al. 2004), show clear spectral variations: the one obtained in 2002 appears featureless, while the one acquired on 2001 clearly shows absorption features at $0.5,0.6-0.65,0.7$ and $0.8-0.9 \mu \mathrm{m}$, very similar to those found in several main belt asteroids believed to have undergone aqueous alteration, and tentatively attributed to aqueous altered minerals.

A few TNOs appear to show spectral features related to the presence of aqueous altered materials on their surfaces (Fornasier et al. 2009). Aqueous alteration is a low temperature chemical alteration of materials which takes place in presence of liquid water. Its detection might indicate the presence of water ice in the original bodies, that were enough heated during their early evolutionary phases to develop the alteration at their surface. Hydrous altered materials seem to be present in comets, and hydrous silicates have been detected in interplanetary dust particles and micrometeorites. As suggested by Jarvis \& Vilas (2000), if the presence of spectral characteristics usually related to aqueous alteration processes is confirmed on the surface of TNOs, a mechanism to produce them even at large heliocentric distances must be defined. How aqueous alteration could have occurred at such low temperatures far from the Sun is not well understood, but it cannot be excluded that hydrated minerals could have been formed directly in the early solar nebula. Large TNOs may have been subjected to significant radiogenic heating 


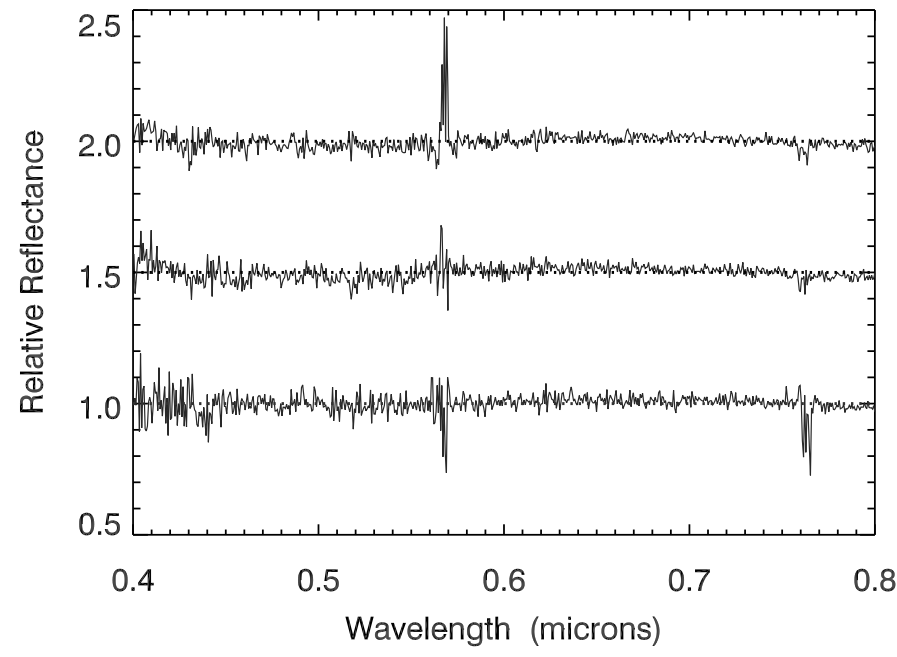

Fig. 3. Three reflectance spectra of Huya in the visible, all corrected to their spectral slope and all normalized at $0.55 \mu \mathrm{m}$. Spectra have been shifted by $0,0.5$ and 1 unit, for clarity. Spectra appear chronologically from bottom to the top. In each case, the horizontal dot-line represents the normalized reflectance value.

(Luu \& Jewitt 2002), but it remains to be seen if this would have been sufficient for aqueous alteration of the anhydrous material inside the TNOs, and also a mechanism capable of transporting enough heat to the surface would be required. Another way to heat a TNO is through impacts, which could have provided a transient heat source. However, the heat generated and the duration of the heating episode(s) may not have been sufficient for aqueous alteration of minerals (e.g., Kerridge \& Bunch 1979).

The three new spectra presented in this paper have quite similar behavior in the entire vis-nIR wavelength range. The results are consistent with the presence of a shallow feature near $2.0 \mu \mathrm{m}$ and flux decreases beyond $1.9 \mu \mathrm{m}$ on all the three spectra, confirming previous results made in the nIR. This could suggest the probable presence of small amount of water ice on the whole surface of this object, or that a few patches of water ice are quite homogeneously distributed on the entire surface of Huya. However, we can not confirm the presence of clear absorption features in the visible range (absorption depth lower than $2 \%$ at best, see Fig. 3). The three spectra are featureless between 0.6 and $0.9 \mu \mathrm{m}$ and we expect that the surface observed during these three attempts was mainly devoid of any content related to aqueous alteration processes. Thirouin et al. (2014) recently performed visible photometry to constrain the rotational period of this object and found different possibilities from their periodogram. Their best solution seems to be in agreement with a rotational period of $5.28 \mathrm{~h}$, but three other peaks have been obtained and can not be ruled out. These other results correspond to rotational periods of $4.31,6.63$ and $9.15 \mathrm{~h}$. In all cases, the corresponding singlepeaked light curve amplitude is $0.02 \pm 0.01 \mathrm{mag}$ only, and authors warn on the difficulty to estimate a secure rotational period among these four possibilities.

Since we observed Huya three times in a short time span, we can test the different periods to check if: a) all our spectroscopic observations cover the whole surface of Huya and then, we can quite logically rule out the presence of any material related to aqueous alteration or, b) if a fraction of the surface was not well covered (or not at all), we could suggest that this material, previously expected by Lazzarin et al. (2003) from their observations, could be localized in this part of the surface. We report in Fig. 4 the level of surface coverage for any relative rotational

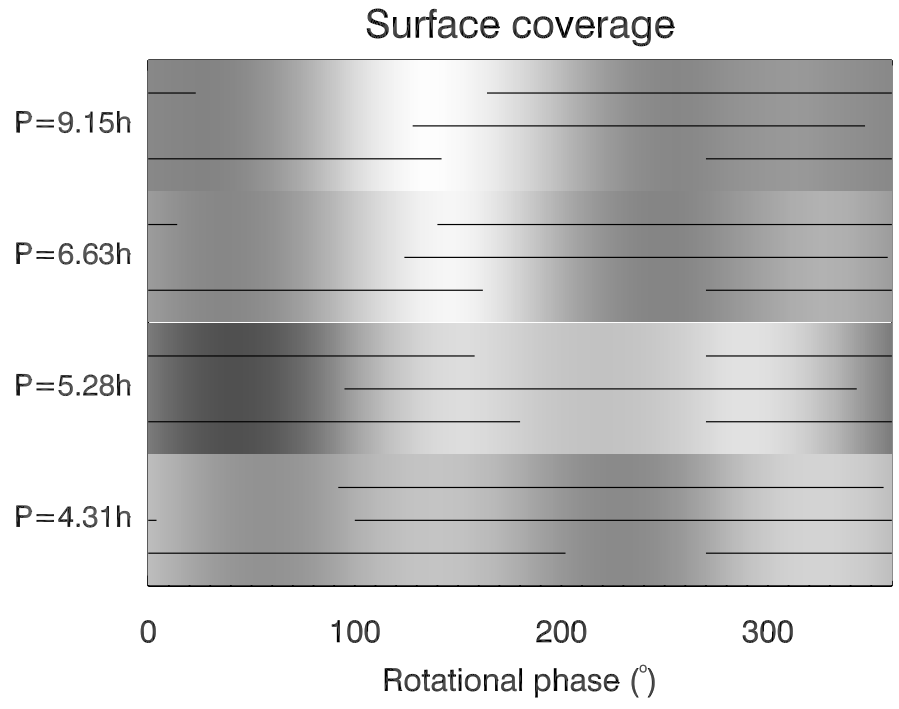

Fig. 4. Surface coverage from the three observations. The grayscale informs on the occurence of observation (surface always observed at subearth time if in black, never observed if in white) as a function of the rotational phase, according to each possible rotational period $(4.31,5.28$, 6.63 and $9.15 \mathrm{~h}$, from bottom to the top). For each rotational period, horizontal lines represent the coverage by night (nightly coverages appear chronologically from bottom to the top). See text for more explanation.

phase, considering the four possible rotational periods obtained by Thirouin et al. (2014). For that, we considered that the $0^{\circ}$ corresponds to the longitude of the sub-earth point at the beginning of the first acquisition performed on 2013-07-13 and we decompose the sphere of Huya as 360 individual longitudinal-units. We also assumed that the contribution of each spatial unit of the surface is proportional to $\cos \theta \cdot \theta$ is the angle formed between the ray of light reflected toward the observer (assumed to be also the direction of the incident light since we consider our observations performed at zero phase angle) and the normal of the center of each unit. Until now, we did not know the pole axis orientation, but our goal here is only to search for possible patches of the surface that could be uncovered during our observations. In addition to that, over its 13 yr Huya has covered less than $5 \%$ of its orbit, corresponding to the delay between our observations and those revealing absorption features in the visible range. Then, we can expect that viewing conditions are almost similar and that orientation of Huya spin axis has not significantly changed. From Fig. 4, we could identify an area that is less covered by our three observations (i.e.: not observed at all or located near the limb at observation time). Considering our rotational phase reference, this area seems to be located near $130-160^{\circ}$. To a lesser extent, we could also consider the area near $290^{\circ}$, considering the best solution with a rotational period of $5.28 \mathrm{~h}$. This result shows that we can not completely rule out the presence of absorption features related to aqueous altered material. But if present, it should be localized in a very small fraction of the surface.

$2007 \mathrm{VH}_{305}$ belongs to the Centaur family. There is no albedo and diameter estimation yet for this object. The visible to near infrared spectrum (see Fig. 2) shows a clear reflectance peak in the $H$ band. This could imply a continuous reddening from 0.4 up to $1.8 \mu \mathrm{m}$ quickly followed by a reflectance decrease in the $K$ band. However, we do not have the $J$ spectrum (i.e., from 1.1 to $1.35 \mu \mathrm{m}$ ) to fully confirm this hypothesis. In addition to that, the visible and near infrared photometry, used to merge both spectral parts, has been performed one day apart. As such 
result on the global shape of the spectrum is quite uncommon among TNOs and Centaurs spectra, we can suspect that the $V J$ color can be significantly different than that obtained, meaning this object is probably elongated and/or heterogeneous. However, the nIR spectral behavior obtained in spectroscopy, with a blue spectral slope in the [1.5-2.4] $\mu \mathrm{m}$ range, is fully consistent with the $H K$ measurement performed in photometry. Further investigation, with new spectroscopic data, are needed in order to identify the chemical content that can be responsible to this spectral properties.

\section{Data set}

We collected spectra of 43 different objects, see Table 2. The data set is composed of 6 objects belonging to the BB group, 17 to the BR group, 9 to the IR group and 11 to the RR group. For each object, we report its complete full name, the delay between the acquisition of the photometry in the visible range and that in the nIR range, as well as the delay between the acquisition of the photometric and spectroscopic data. We also report the dynamical group, the taxonomical group and the adopted colors, found from the literature. Some of the objects have been classified in several taxonomical groups. This is mostly due to the fact that photometrical errors (taken at $3 \sigma$ in Perna et al. 2010) could give alternative and, less frequently, due to real photometric variations. In such cases, we chose the taxonomical group in agreement with the adopted photometry, comparing with the taxonomical mean values presented in Perna et al. (2010). The visible and nIR photometry provide the colors needed to compute the reflectance values in each $R, I, J, H$ and $K$ ranges. Computation of the different reflectances has been made using solar indices presented in DeMeo et al. (2009). Reflectances are also needed to combine the different parts of the spectra, mainly obtained using FORS2, ISAAC and SINFONI in the visible, $J$ and $H+K$ ranges, respectively. In the case of the objects (38628) Huya and (278631) $2007 \mathrm{JJ}_{43}$, the entire vis-nIR spectra have been acquired simultaneously on the same ship with X-shooter, and the photometry is not mandatory to merge the different parts of the spectra.

\subsection{Limits of the data set}

As presented in Table 2, just over a quarter of the objects have been observed contiguously or simultaneously in both visible and nIR ranges with both technics (photometry and spectroscopy). For these objects, we can assume that the vis-nIR data scan the same part of the surface. A little bit more than one another quarter of the data set is mainly composed of objects for which all the data have been acquired within one day. For this sample, contiguous acquisition have been performed between both photometric and spectroscopic technics. For these objects, we can assume almost similar observational circumstances and good quality of the combination of the different nIR spectroscopic parts. However, we can not guaranty a good match between the visible and the nIR parts, especially in case of elongated objects and/or heterogeneous surfaces. For the rest (just over one third of the data set), there are temporal discrepancies between all technics of acquisition and elongated or heterogenous objects as well as variation in observational circumstances (change in the earth-object distance and phase angle, for instance) could led to possible mismatches during the combination procedure of the different parts of the spectra.

\subsection{Elongated shape and heterogeneous objets}

As stated in the previous subsection, our methods to generate full vis-nIR spectra can suffer from elongated shapes or/and the heterogeneity of the surface when data have not been acquired contiguously or simultaneously. In this case, spectral (i.e.: color) variation could indeed appear and not be corrected in case of homogeneous but elongated objects. Physically, an homogeneous and elongated object exhibits similar colors during its entire rotation but generate flux variation in each individual filter as seen from earth. Acquiring photometry in the visible and in the nIR at different rotation phase leads, subsequently, to false vis-nIR color variation. Variation could also physically appear for heterogeneous objects (elongated or spherical). We clearly identify four objects for which this problem occurs since heterogeneity has been reported in literature (objects $N=2,4,12$ and 19 in Table 2). Change in the attribution of the taxonomical group due to color variation can also inform us of this possibility, but these are very few.

\section{Final results}

As the number of objects in each taxonomical class is quite limited, we include all the objects presented in Table 2 to maximize the statistics, as much as possible. We caution that refinement in the establishment of the four mean taxonomical spectra is possible as soon as new data, and especially simultaneous ones, will be accessible. We also add that a few objects have not been included in this study even if complete vis-nIR spectra and taxonomy exist for them. This mainly concerns the biggest and brightest TNOs (such as Makemake and Haumea) or the dwarf planets Eris and Pluto for which specific strong absorption features have been detected and could be associated to very young and fresh surfaces implying sublimation and redeposition processes that could only occur on big and distant objects.

In order to average the spectra of all the objects, for a given taxonomical group, we first computed a common wavelength vector at the spectral resolution of 250 between 0.45 and $2.40 \mu \mathrm{m}$. We then convolved each spectrum, obtained at various spectral resolution, with this common vector and compute the error at each wavelength as well. The error in each initial individual spectrum, before resampling, was computed as a combination of the spectroscopic local standard deviation (computed from the 20 closest neighbors, in the wavelength direction) and the photometric error (converted into reflectance errors) for the $J, H$ and $K$ ranges. We then obtained a reflectance spectrum and its error at a spectral resolution of 250 for each object. In order to compute the final spectra, one for each taxonomical group, and the corresponding errors, we used a Monte Carlo approach. For each object, we generated 1000 synthetic spectra where the reflectance at each wavelength was randomly fixed in the $\pm 1 \sigma$ interval. The reflectance value of each group at each wavelength has been computed as the mean value of all the synthetic spectra of all the $\mathrm{N}_{i}$ objects included in the defined $i^{\text {nth }}$ taxonomical group while the final error has been computed at $1 \sigma$ level from the $N_{i} \times 1000$ spectra. All the four averaged spectra are presented in Fig. 5.

Our results appear to be, at first order, very consistent with the mean reflectance obtained from photometry on a bigger data set (composed of 151 Centaurs and TNOs). We clearly see good agreement for both BB and RR groups, with mean value and errors almost similar within the entire wavelength range. In details, however, we see that results are a little worse for the BR and IR groups. Even if the results are still in good agreement within the 


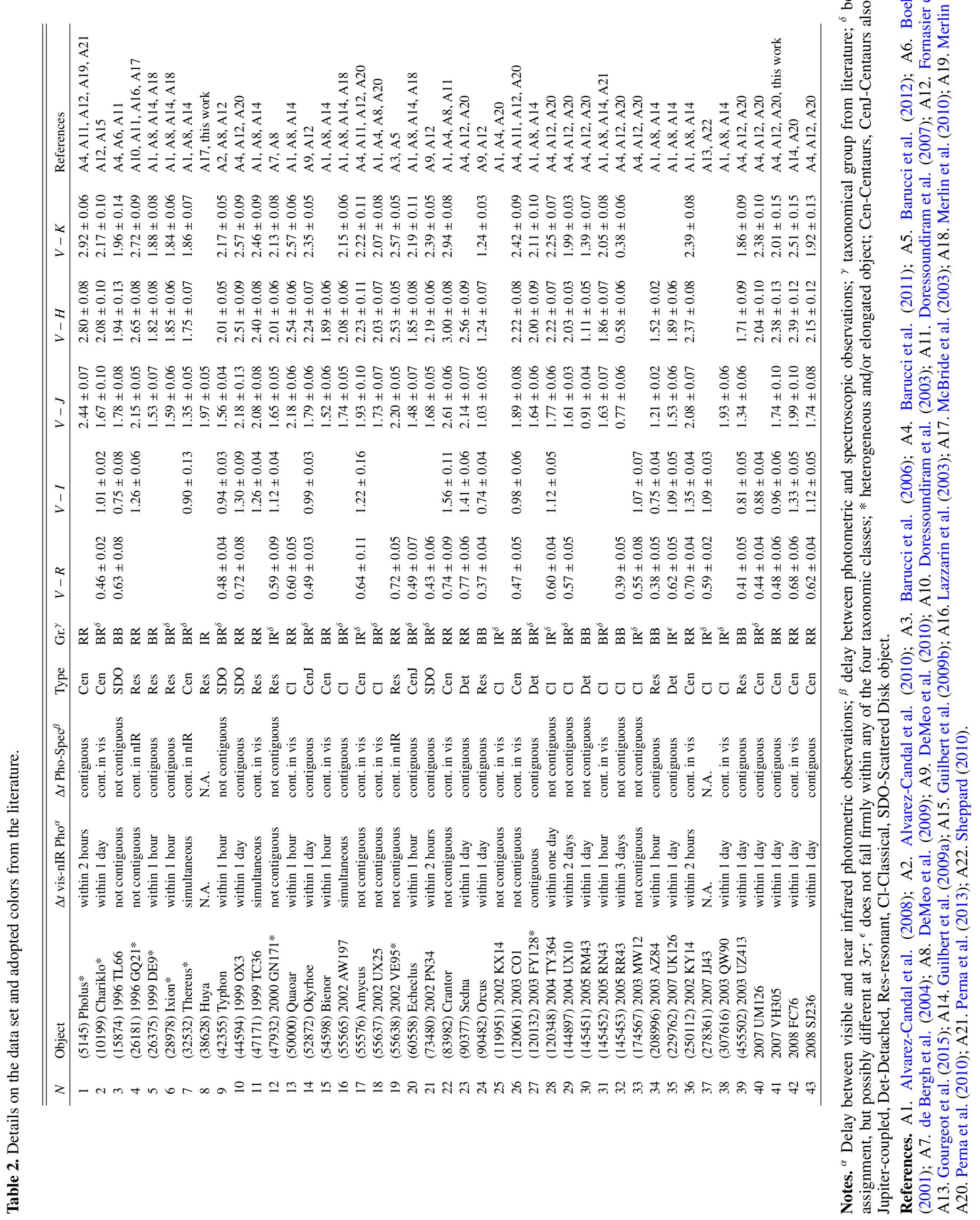


F. Merlin et al.: Taxonomy of trans-Neptunian objects and Centaurs as seen from spectroscopy

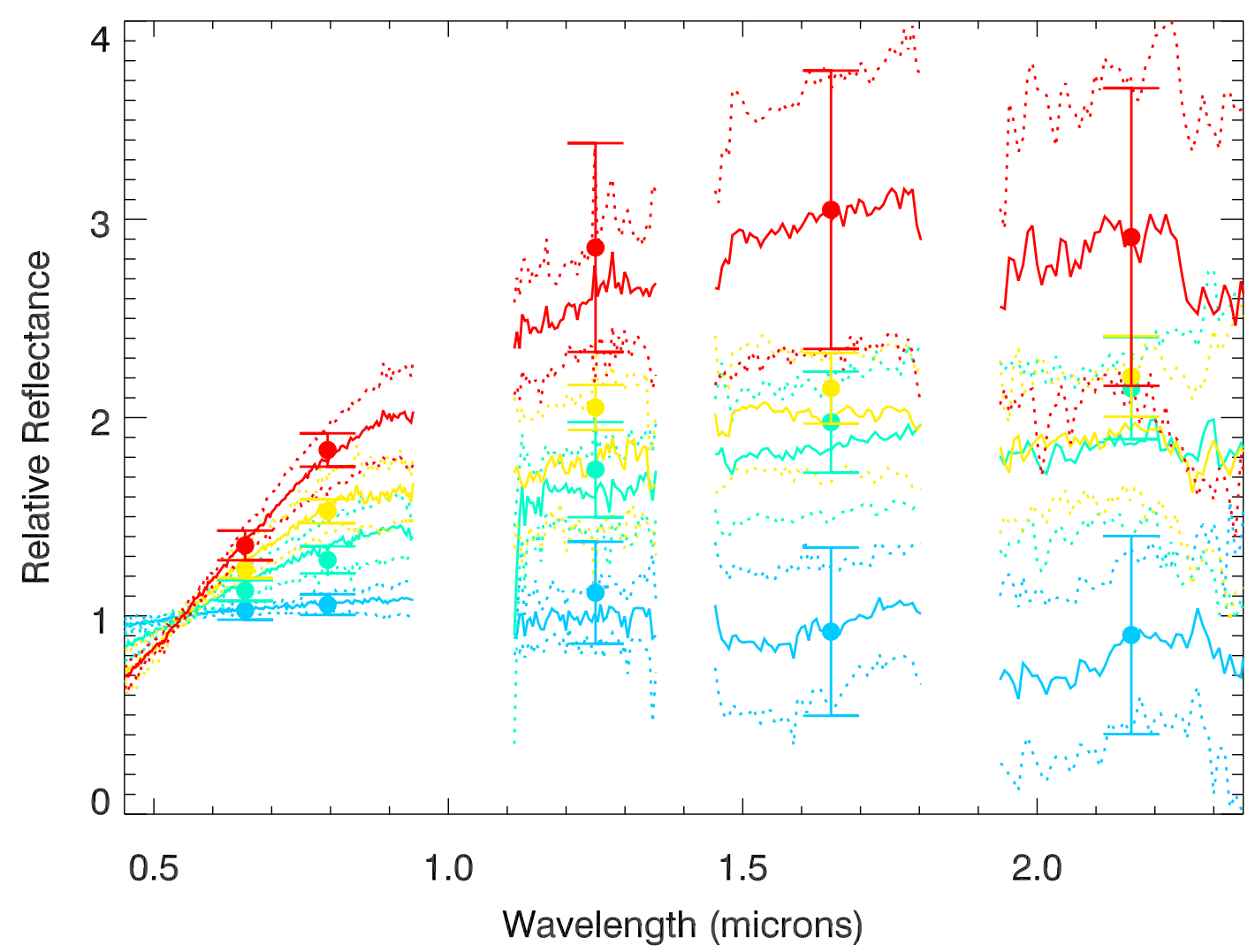

Fig. 5. Four mean spectra, normalized at $0.55 \mu \mathrm{m}$. The spectra, from bottom to the top, are in blue, green, yellow and red and correspond to BB, BR, IR and RR taxonomical classes, respectively. The error in dashed line appear at $\pm 1 \sigma$ level, see text for more explanation on errors determination. The photometrical data (centered at $0.655,0.795,1.25,1.65$ and $2.165 \mu \mathrm{m}$ ) giving the average reflectance values for each taxon are taken from Perna et al. (2010).

errors, the reflectance of the spectroscopic measurement is systematically in the low limit of that obtained in photometry in the near infrared (i.e., in $J, H$ and $K$ photometric ranges). This disagreement could be due to the fact that our spectroscopic sample is smaller than that used in photometry, even if this statement seems to be not compatible with the fact that the BR group is the one composed of the greatest number of objects. One another possibility is also that most part of the BR and IR objects could be tentatively assigned in other taxonomical groups (see Perna et al. 2010, for more details). In all cases, the four mean spectra are accurate enough to be used to compute reflectances in different filter systems in the $[0.45-2.40] \mu \mathrm{m}$ range. The gaps located near 1 and $1.4 \mu \mathrm{m}$ are not too broad, and the lack of expected absorption features in these wavelength ranges could authorized to fill these wavelength ranges in the context of spatial instruments. The gap near $1.9 \mu \mathrm{m}$ should be taken with caution instead, since the pattern of the deepest absorption feature of the water ice occurs at this wavelength within the entire [0.45$2.40] \mu \mathrm{m}$ range. Moreover, the presence of this absorption feature is quite obvious on the mean spectrum of the BB group and that of the RR group. From the spectral behavior of the mean taxonomical spectra (and more especially from the depth of the absorption features located near 1.5 and $2.0 \mu \mathrm{m}$, see Fig. 5), we can also almost confirm results published by Barucci et al. (2008a) on the fact that the water ice content increases within the four taxonomical classes in the following order IR, BR, RR and BB. We find absorption band depth of $5.3 \pm 0.9 \%, 5.2 \pm 0.5 \%$, $7.9 \pm 1.4 \%$ and $31.4 \pm 4.6 \%$, for the IR, BR, RR and BB mean spectra, respectively. To a lesser extent, we can also mention the presence of an absorption feature near $2.3 \mu \mathrm{m}$ for the RR and IR groups only, finding an absorption band depth of $14.4 \pm 4.9 \%$ and $9.9 \pm 3.7 \%$, respectively. This band could be an indicator of the presence of hydrocarbons and/or methanol, as supported by Dalle Ore et al. (2015) to explain the composition of the ultra-red TNOs. We still used a Monte Carlo approach to obtain these results. In detail, we generated 1000 synthetic spectra for each taxonomical mean spectrum (where the reflectance at each wavelength was randomly fixed within $1 \sigma$ level) and ran Gaussian fits in the [1.72-2.18] $\mu \mathrm{m}$ and [2.18-2.38] $\mu \mathrm{m}$ ranges for each of them. All the fits have been computed using a common IDL procedure (MPFIT) and assuming a band centered in the [1.97-2.03] $\mu \mathrm{m}$ range in the case of water ice, and in the [2.27-2.32] $\mu \mathrm{m}$ range in the case of hydrocarbons.

\section{Conclusions}

Several new vis-nIR spectra of two objects have been presented in this work. No clear and strong absorption feature has been detected for either of them in the complete [0.45-2.40] $\mu \mathrm{m}$ range. From our investigations:

1. We conclude that the three observations of Huya cover most of the entire surface of this TNO, according to the possible rotational periods. At first order, all the three spectra are quite similar, with equal red slope in the visible and neutral slope in the nIR.

2. We do not detect any absorption feature on Huya, tentatively attributed to aqueous altered minerals by Lazzarin et al. (2003) in 2001, and detected during one unique observation. We identified a few possible longitudinal-areas where this material could be possibly detected, if truly present. 
3. Our observations are consistent with a shallow absorption feature near $2.0 \mu \mathrm{m}$, attributable to the presence of water ice, already reported by several authors. This feature appears on all the three spectra of Huya, and we suspect that Huya is homogeneously covered by a few amount of $\mathrm{H}_{2} \mathrm{O}$.

4. The spectrum of the Centaur $2007 \mathrm{VH}_{305}$ shows a moderate red slope in the visible and a strong blue slope in the HK range, fully compatible with photometric data. Further investigation are needed to fully understand the reasons of this spectral behavior.

Then, we added these spectra in a large spectroscopic data set of 43 objects in total including twelve Centaurs, two other Centaurs also classified as Jupiter-coupled object by Gladman et al. (2008) and likely more evolved than the other Centaurs, ten resonant objects, four scattered disk objects, eleven classical objects and four detached objects. From this database:

1. We generated four vis-nIR spectra corresponding to the mean spectra of the four taxonomical groups BB, BR, IR and RR.

2. We primarily confirm the spectroscopic trend found by Barucci et al. (2008a) on the occurence of $\mathrm{H}_{2} \mathrm{O}$ within the four taxonomical classes. The ice content increases in the following order IR and BR (the results are quite similar for these two groups), RR, then BB.

3. We showed that the RR and IR mean spectra exhibit an absorption feature at wavelength above about $2.3 \mu \mathrm{m}$, that could be an indicator of the occurence of hydrocarbons and/or methanol at the surface of these ultra-red objects, as presented by Dalle Ore et al. (2015).

Acknowledgements. We thank the anonymous referee for their suggestions. This project was supported by the French Planetology National Programme (INSUPNP). A. Alvarez-Candal thanks CNPq and FAPERJ for support through diverse grants and fellowships.

\section{References}

Alvarez-Candal, A., Barucci, M. A., Merlin, F., Guilbert, A., \& de Bergh, C. 2007, A\&A, 475, 369

Alvarez-Candal, A., Fornasier, S., Barucci, M. A., de Bergh, C., \& Merlin, F. 2008, A\&A, 487, 741

Alvarez-Candal, A., Barucci, M. A., Merlin, F., et al. 2010, A\&A, 511, A35

Barucci, M. A., Capria, M. T., Coradini, A., \& Fulchignoni, M. 1987, Icarus, 72, 304
Barucci, M. A., Belskaya, I. N., Fulchignoni, M., \& Birlan, M. 2005, AJ, 130, 1291

Barucci, M. A., Merlin, F., Dotto, E., Doressoundiram, A., \& de Bergh, C. 2006, A\&A, 455, 725

Barucci, M. A., Boehnhardt, H., Cruikshank, D. P., \& Morbidelli, A. 2008a, in The Solar System Beyond Neptune, eds. M. A. Barucci, H. Boehnhardt, D. P. Cruikshank, A. Morbidelli, \& R. Dotson, 3

Barucci, M. A., Brown, M. E., Emery, J. P., \& Merlin, F. 2008b, in The Solar System Beyond Neptune, eds. M. A. Barucci, H. Boehnhardt, D. P. Cruikshank, A. Morbidelli, \& R. Dotson, 143

Barucci, M. A., Alvarez-Candal, A., Merlin, F., et al. 2011, Icarus, 214, 297

Barucci, M. A., Merlin, F., Perna, D., et al. 2012, A\&A, 539, A152

Boehnhardt, H., Tozzi, G. P., Birklei, K., et al. 2001, A\&A, 378, 653

Brown, M. E., Blake, G. A., \& Kessler, J. E. 2000, ApJ, 543, L163

Dalle Ore, C. M., Barucci, M. A., Emery, J. P., et al. 2015, Icarus, 252, 311

de Bergh, C., Boehnhardt, H., Barucci, M. A., et al. 2004, A\&A, 416, 791

DeMeo, F. E., Fornasier, S., Barucci, M. A., et al. 2009, A\&A, 493, 283

DeMeo, F. E., Barucci, M. A., Merlin, F., et al. 2010, A\&A, 521, A35

Doressoundiram, A., Tozzi, G. P., Barucci, M. A., Boehnhardt, H., \& Fornasier, S. Romon, J. 2003, AJ, 125, 2721

Doressoundiram, A., Peixinho, N., Moullet, A., et al. 2007, AJ, 134, 2186

Doressoundiram, A., Boehnhardt, H., Tegler, S. C., \& Trujillo, C. 2008, in The Solar System Beyond Neptune, eds. M. A. Barucci, H. Boehnhardt, D. P. Cruikshank, A. Morbidelli, \& R. Dotson, 91

Fornasier, S., Barucci, M. A., de Bergh, C., et al. 2009, A\&A, 508, 457

Fornasier, S., Lellouch, E., Müller, T., et al. 2013, A\&A, 555, A15

Fulchignoni, M., Belskaya, I., Barucci, M. A., de Sanctis, M. C., \& Doressoundiram, A. 2008, in The Solar System Beyond Neptune, eds. M. A. Barucci, H. Boehnhardt, D. P. Cruikshank, A. Morbidelli, \& R. Dotson, 181

Gladman, B., Marsden, B. G., \& Vanlaerhoven, C. 2008, in The Solar System Beyond Neptune, eds. M. A. Barucci, H. Boehnhardt, D. P. Cruikshank, A. Morbidelli, \& R. Dotson, 43

Gourgeot, F., Barucci, M. A., Alvarez-Candal, A., et al. 2015, A\&A, 582, A13

Guilbert, A., Alvarez-Candal, A., Merlin, F., et al. 2009a, Icarus, 201, 272

Guilbert, A., Barucci, M. A., Brunetto, R., et al. 2009b, Icarus, 501, 777

Jarvis, K. S., \& Vilas, F. 2000, in AAS/Division for Planetary Sciences Meeting Abstracts \#32, BAAS, 32, 1641

Jewitt, D. C., \& Luu, J. X. 2001, AJ, 122, 2099

Kerridge, J. F., \& Bunch, T. E. 1979, in Asteroids, ed. T. Gehrels, 745

Lazzarin, M., Barucci, M. A., Boehnhardt, H., et al. 2003, AJ, 125, 1554

Licandro, J., Oliva, E., \& Di Martino, M. 2001, A\&A, 373, L29

Luu, J. X., \& Jewitt, D. C. 2002, ARA\&A, 40, 63

McBride, N., Green, S., Davies, J. K., et al. 2003, Icarus, 161, 501

Merlin, F., Barucci, M. A., de Bergh, C., et al. 2010, Icarus, 208, 945

Merlin, F., Quirico, E., Barucci, M. A., \& de Bergh, C. 2012, A\&A, 544, A20

Noll, K. S., Grundy, W. M., Schlichting, H., Murray-Clay, R., \& Benecchi, S. D. 2012, IAU Circ., 9253

Perna, D., Barucci, M. A., Fornasier, S., et al. 2010, A\&A, 510, A53

Perna, D., Dotto, E., Barucci, M. A., et al. 2013, A\&A, 554, A49

Sheppard, S. S. 2010, AJ, 139, 1394

Thirouin, A., Noll, K. S., Ortiz, J. L., \& Morales, N. 2014, A\&A, 569, A3 